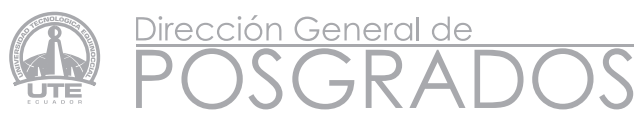

EíDOS

\section{PROPUESTA PARA LA INTERNACIONALIZACIÓN DE LA UNIVERSIDAD ECUATORIANA EN EL ÁMBITO DE PROYECTOS DE INVESTIGACIÓN - ENSAYO DESCRIPTIVO}

1Martha Concepción Macías Sánchez, ${ }^{2}$ Víctor Hugo Abril Porras, ${ }^{3}$ Javier Hernando Sanmartín Rojas

\begin{abstract}
1Universidad de las Fueerzas Armadas. ESPE. Sangolqui -Ecuador. marthadeeguez@yahoo.com / mcmacias3@yahoo.com.

2Universidad Tecnológica Equinoccial / Universidad de las Fuerzas Armadas ESPE. Quito-Ecuador. victor. abril@ute.edu.ec / vhabril@espe.edu.ec
\end{abstract}

${ }^{3}$ Universidad de las Fuerza Armadas-ESPE. Sangolquí-Ecuador. jhsanmartin@espe.edu.ec

Recepción / Received: 24,08,2015

Aceptación / Accepted: 18,11,2015

Publicado / Published: 30,12,2015

\section{Resumen:}

Este artículo tiene como finalidad aportar con una propuesta de internacionalización de la universidad ecuatoriana en el ámbito de proyectos de investigación, considerando que la globalización y el conocimiento no tienen fronteras, su repercusión y aporte a la sociedad contribuye a otorgarle una mejor calidad de vida, que se traduce en lograr seres humanos que coadyuvan a mejorar el desarrollo en cada uno de los entornos en los que se desenvuelven. El objetivo de esta propuesta es plantear estrategias para tener Instituciones de Educación Superior (IES), que incluyen a universidades y escuelas politécnicas, con proyección internacional y que estén inmersas en proyectos y procesos de investigación, nutridos de experiencias y conocimiento que aporten al beneficio del mejoramiento de la sociedad ecuatoriana. Este beneficio se da través de la formación e investigación, viabilizadas por la realización de convenios, suscripciones, conformación de redes internacionales, formación de docentes en ámbitos de investigación con altos estándares de calidad, que permitan proyectar a la universidad ecuatoriana a formar parte de los ranking internacionales que miden la producción científica e investigación, que generan prestigio y posicionamiento, pero sobre todo que dicho conocimiento y su gestión aporten al desarrollo de la sociedad. Lo dicho anteriormente se plantea como un reto, para lo cual se propone estrategias, cuya responsabilidad se encuentra en el Estado y en cada una de las IES, que deben trabajar colaborativamente para el éxito de su implementación.

Palabras clave: Internacionalización, universidad, estrategias, investigación, desarrollo, proyecto de investigación.

\begin{abstract}
:
Globalization and knowledge have no frontiers. The objective of this article is to develop a proposal for the internationalization of Ecuadorian universities research projects. The aim of this proposal is to achieve universities with international exposure and immersed on research processes. These will nurture them with experiences and knowledge in the benefit of society. Such benefit is crystalized through education and research, which is feasible with agreements, subscriptions, international networking, and formation of researchers that could step up Ecuadorian universities into international rankings in regards to scientific production and research. These rankings improve prestige and positioning. The internationalization of Ecuadorian universities' research projects is a challenge that requires joint strategies. The universities and the State are both responsible for these strategies and should work in collaboration for a successful implementation.
\end{abstract}

Keywords: Internationalization, university, strategies, research, development, research projects 


\section{INTRODUCCIÓN}

La internacionalización de la universidad ecuatoriana, para este estudio entiéndase universidades y escuelas politécnicas, es el proceso de integrar la dimensión internacional, y global para proveer la enseñanza, la investigación y los servicios universitarios, siendo estos tres factores, sustanciales en el quehacer universitario.

De acuerdo a la Organización de las Naciones Unidas para la Educación, la Ciencia y la Cultura (UNESCO) en la "Declaración Mundial sobre educación superior para el siglo XXI: Visión y Acción (1998) se afirma que la calidad requiere que la educación superior se caracterice por su dimensión internacional" (UNESCO, 1998). Estas dos manifestaciones ratifican la importancia de la internacionalización de la educación superior para que el conocimiento pueda trascender sin fronteras, que es el caso de la investigación.

Vigil Taquechel (2013) afirma que existen estereotipos, que reducen la internacionalización de la educación a acciones de movilidad docente y estudiantil, en detrimento de muchas otras manifestaciones, que pueden ser implementadas a nivel internacional en el seno de una institución universitaria con los más variados objetivos: mejorar el prestigio y visibilidad, fortalecer la capacidad institucional, mejorar la calidad de la docencia y la investigación, contribuir al desarrollo económico local o regional, aportar a la producción de conocimiento o generar ingresos. Por lo que, es importante identificar las oportunidades y los retos que tiene la universidad ecuatoriana, frente a esta coyuntura que se presenta en el marco de una integración regional.

La globalización y la competitividad, han ocasionado que la sociedad reflexione sobre la articulación en varios ámbitos, y la educación no se puede quedar fuera de esta tendencia, tomando en cuenta que su presencia genera resultados con un "efecto mariposa" en el entorno. De aquí la importancia de que las universidades asuman su responsabilidad de globalizar el conocimiento a través de aspectos diversos como aportes científicos, accesibilidad a la tecnología, abaratamiento y/o masificación de servicios, entre los más importantes.
La internacionalización contribuye a un proceso de integración mundial, donde la educación juega un rol central e importante en el progreso de las sociedades, en sus escenarios sociales, culturales, económicos, independiente de las dinámicas locales; por lo tanto, es necesario un cambio en la cultura interna y como sistema de educación superior, convirtiéndose en una necesidad la apertura hacia el contexto internacional en el ámbito investigativo.

\section{LA PROBLEMÁTICA}

En el año 2013, las universidades ecuatorianas fueron evaluadas en lo que concierne a la calidad institucional, el modelo contempló cinco criterios, siendo éstos academia, gestión institucional, docencia, infraestructura e investigación. Uno de los parámetros que registró menor calificación a nivel de todo el sistema, (0.2/1.0, es decir apenas el $20 \%$, y el estándar es por lo menos equivalente al $60 \%$ ), fue el de producción científica e investigación, ya que en el Ecuador no ha existido una cultura investigativa, en vista de que el rol de la universidad se había centrado solo en la formación, olvidando que también es parte del quehacer universitario la generación de conocimiento a través de la investigación.

Al no ser haber sido una prioridad la investigación, tampoco había una conciencia de preparar a los docentes y estudiantes en metodologías de investigación, menos aún de capacitarlos para que estén preparados generar conocimiento en otros idiomas, es decir se carecía de iniciativas de generación de proyectos de investigación y no existía el talento humano formado para crear y gestionar ese conocimiento, por ende tampoco se asignaban recursos financieros para tal objetivo.

El Dr. Luis Romo Saltos, científico ecuatoriano, manifestaba en forma reiterada que creía en el Ecuador, en sus recursos, pero sobre todo en la capacidad de las personas y decía: "Una condición sine qua non para asegurar el éxito de la investigación es el mantenimiento ineludible de la continuidad de los trabajos de investigación que se debe garantizar asegurando la estabilidad de las respectivas masas críticas y la disponibilidad de fondos, con lo cual son dos factores importantes para la producción científica: el talento humano y el financiamiento" (Romo Saltos, 2004). De ahí la aseveración de las universidades que manifiestan entre otros, estos dos factores como limitantes para esta actividad. 
Entre las razones que dan las universidades para no tener mayor producción e investigación científica es la falta de recursos, la falta de formación de los docentes en investigación, y la decisión de las autoridades de concebirse como universidades de docencia y no de investigación, sin tener clara conciencia de que la investigación es lo que permite generar nuevo conocimiento, y por ende soluciones a los problemas de la sociedad; y, a nivel internacional le permite a la institución entrar en el debate del state of the art en las diferentes disciplinas, que le otorgan un mejor posicionamiento global.

Según Samaniego, rector de la Universidad Central del Ecuador, y Gangotena, rector de la Universidad San Francisco de Quito reconocen que la investigación es el talón de Aquiles histórico de la universidad local, y afirman que existe una falta de masa crítica que permita el diálogo científico, además añade que "Un físico en Estados Unidos de América publica en un año lo mismo que una universidad ecuatoriana, en todas sus áreas, en cinco años". De acuerdo a datos del Banco Mundial (2014), el Ecuador no registra investigadores por cada millón de habitantes en el período 2010 2014 a nivel internacional.

El Consejo de Evaluación, Acreditación y Aseguramiento de la Calidad de la Educación Superior (CEAACES, 2013), tomó cuatro criterios para evaluar el nivel de las universidades al generar nuevo conocimiento a través de la investigación: planificación, investigación regional, producción científica y libros revisados por pares.

Según el informe de SCImago Research Group, manejado por cuatro universidades de España y que se basa en el sistema Scopus para medir la publicación internacional de universidades, la USFQ publicó 352 artículos en revistas indexadas entre el 2007 y 2011; la Pontificia Universidad Católica del Ecuador - 247; la Escuela Politécnica Nacional - 136; la Escuela Politécnica del Litoral - 83; y, la Universidad Central del Ecuador - 77. Pero la producción investigativa respecto del contexto internacional es reducida. Según el indicador SRJ (SCImago Journal Rank), Ecuador en el año 2014 está en el puesto 12 entre 47 países de Latinoamérica con una producción de 564 documentos.

Es importante tomar en cuenta el criterio de la UNESCO, de crear un ranking adecuado a las necesidades de Latinoamérica para medir el desempeño de las universidades en un contexto más acorde a la propia realidad, pero sin descuidar la visión global, de que las universidades generadoras de conocimiento tienen mejor infraestructura, mayor inversión en investigación y un claustro académico formado en investigación, lo que se convierte también en un reto, tanto para el Estado, como para las IES ecuatorianas, ya que estos factores forman parte de sus limitaciones.

De aquí se desprende que para formar parte de los rankings de desempeño a nivel internacional (en lo que corresponde a Latinoamérica), falta aún hacer mayor esfuerzo por asignar las inversiones correspondientes a las áreas que califican los criterios que se vuelven atractivos para generar una oferta académica a nivel internacional. Altbach and Knight (2007), al analizar las universidades de clase mundial, subraya que su principal característica es que se centran en los procesos de investigación y desarrollo, los que requieren de una inversión alta para su financiamiento.

En el Ecuador la Secretaría Nacional de Educación Superior, Ciencia, Tecnología e Innovación (SENESCYT) está llevando a cabo un proyecto denominado "Prometeo" que consiste en atraer a investigadores internacionales con grado académico de doctorado equivalente a Ph.D. y se los vincula con diferentes instituciones para que aporten con proyectos que den soluciones relacionadas con el Plan de Desarrollo del país, pero a la fecha se evidencia que requiere mayor impulso para obtener resultados tangibles.

Mencionada la problemática y el diagnóstico en la falta de producción científica y de investigación en el Ecuador, se hace necesaria la incursión urgente en una cultura investigativa, con su inminente internacionalización para que el conocimiento realmente traspase fronteras en forma bilateral: que los investigadores nacionales se nutran de conocimientos foráneos y viceversa, para sumar saberes, para generar y gestionar el conocimiento.

Para el sistema de educación superior en Ecuador, dada la tendencia de trabajar bajo un contexto local, con una visión global, se convierte en un reto la búsqueda de relacionamiento con los ámbitos internacionales a fin de responder a demandas sociales, donde es importante incursionar en la experiencia de la internacionalización, que aporta con múltiples beneficios como es la transferencia de conocimiento que enriquecen y coadyuva al mejoramiento de la calidad de vida de nuestra sociedad.

El 30 de junio del 2013, se realizó en Quito el primer taller de "Políticas Públicas y Modelos Universitarios" impulsado por la SENESCYT, donde concluyeron que uno de los grandes problemas a tratar es la falta de investigación, y la necesidad de establecer políticas al respecto. 
El desarrollo de investigación es parte del quehacer universitario, la generación de conocimiento nuevo es parte fundamental de la cátedra de todo profesor, por consiguiente es vital que las universidades desarrollen estrategias para fomentar la investigación, para cumplir con su misión y su razón de ser, que es formar y dar soluciones a la sociedad.

\section{Antecedentes normativos en el ámbito de investigación en el Ecuador}

Para que las universidades ecuatorianas incurran en una cultura investigativa, es necesario renovar los currículos y las estructuras académicas, en el caso ecuatoriano el organismo encargado de regular el quehacer universitario es el Conejo de Educación Superior (CES), mismo que realiza los respectivos análisis, socializaciones, conversatorios para llegar a consensos con el sistema universitario, en sus diferentes ámbitos, y entre ellos sobre investigación.

Respecto a la investigación, la legislación ecuatoriana ("Ley Orgánica de Educación Superior," 2010) hace referencia que ésta es parte del quehacer de la universidad, y menciona que ésta debe "fomentar y ejecutar programas de investigación de carácter científico, pedagógico y tecnológico que coadyuve al mejoramiento y protección del medio ambiente y promuevan el desarrollo sustentable nacional", y afirma que el sistema de educación superior deberá "Garantizar la producción de pensamiento y conocimiento articulado con el pensamiento universal".

Es decir desde el marco legal nacional ya se concibe la internacionalización de la investigación, al tomar en cuenta que esta generación de conocimiento debe estar articulado con el pensamiento universal.

\section{Proyectos de investigación}

El proyecto en sí se puede definir como un planteamiento en relación a un problema o necesidad específica que se investiga y se sintetiza en una hipótesis que describe el estudio que realizará el investigador, donde se manifiesta los objetivos y la metodología a utilizar, es decir como lo va a desarrollar; es decir en este caso, el proyecto se convierte en una herramienta indispensable para potenciar la actividad investigativa, en las universidades, lo que lleva a crear o potenciar líneas de investigación, para a partir de éstas plantear programas en los cuales se centrará la producción científica.

Los beneficios del establecimiento de líneas de investigación apoyan a un trabajo más eficiente porque permiten: prever una continuidad y una coherencia en la actividad investigativa universitaria; prever las potenciales investigaciones futuras, dejar abierta la opción de integrar nuevos proyectos y desde perspectivas múltiples, el trabajo con líneas de investigación fomenta la difusión de los informes de investigación en revistas indexadas o de divulgación técnico-científicas que publiquen las universidades o editoriales de prestigio internacional reconocidas por áreas de conocimiento, o como parte de referencias bibliográficas de otras publicaciones, o libros, que con seguridad estarán relacionados con temáticas afines y contribuyendo a la generación de conocimiento.

Y como se evidencia, también desde el ámbito normativo nacional se establecen ya las líneas de investigación que deben ir enlazadas como aporte a solucionar situaciones que plantea el Plan Nacional de Desarrollo, siendo las universidades las llamadas a ser y convertirse en ejes motores de la matriz productiva.

\section{PROPUESTA Y PROCEDIMIENTO PARA LA INTERNACIONALIZACIÓN}

Llegar a generar conocimiento y tener posicionamiento internacional implica tener profesores con experiencia y preparación en investigación, y los recursos necesarios para hacerlo y asociarse en función del expertise de cada uno; y para lograr esto, el primer desafío es fomentar la cultura investigativa en el claustro docente.

En la actualidad, la universidad ecuatoriana enfrenta retos vitales e importantes para caminar hacia la internacionalización. En primer lugar es necesario contar con talento humano, preparados y cualificados para entrar en el círculo investigativo en un entorno global; y la creación de programas, proyectos, redes de investigación y formación con cooperación internacional.

Tomando en cuenta lo que se expone en el IMPI (Indicators for mapping and profiling internationalization), la universidad debe trabajar en cinco dimensiones para incrementar el nivel de internacionalización (Beerkens et al., 2010):

a) Mejorar la calidad de la formación en términos internacionales.

b) Mejorar la calidad de la investigación internacional. 
c) Formar estudiantes para un mundo globalizado.

d) Incrementar la reputación y visibilidad internacional.

e) Proporcionar un servicio a la sociedad y a la comunidad en general.

De estas cinco dimensiones, el presente trabajo se centra en el segundo relacionado con la investigación porque para el Ecuador representa una necesidad inminente de entrar en el círculo investigativo hacia una internacionalización, y para lo cual se plantea la siguiente propuesta:

a) Fomentar la cultura investigativa en el claustro docente

b) Mejorar la cantidad y calidad de la producción investigativa.

c) Implementar estrategias de trabajo investigativo que permitan ajustarse a los indicadores y parámetros de calidad a nivel nacional e internacional

d) Validar los recursos y competencias del sistema de educación superior y de sus miembros (IES), en relación a proyectos de investigación.

e.Incentivar el retorno de los profesionales que están en proceso de formación de doctorado y evitar la fuga de cerebros.

Hay que tomar en cuenta existen limitaciones para la posible implementación de esta propuesta, reconociendo que la universidad ecuatoriana está realizando esfuerzos importantes, pero aún es corto el tiempo para haber superado las falencias detectadas en el 2013, específicamente en el ámbito de la investigación, por lo tanto vencer las siguientes restricciones para el proceso de internacionalización se convierten en un reto a superar de manera urgente:

a) Falta de una cultura hacia la investigación.

b) Falta de investigadores preparados.

c) Falta de formación en investigación, tanto a estudiantes como a docentes.

d) Falta de recursos económicos, y como consecuencia, una carencia de infraestructura y equipamiento de las universidades y sus laboratorios, para la actividad investigativa

e) Las limitaciones en la suficiencia de otros idiomas por parte de la comunidad universitaria en general, especialmente del inglés con mayor información, producto de las investigaciones a nivel mundial.

Es importante superar dichas barreras y trabajar desde el inicio en el cambio de cultura organizacional dentro de las mismas universidades.

El primer paso para fomentar la cultura de investigación, es impulsar un proceso de consensos para crear políticas institucionales, con la participación de alumnos, docentes, autoridades y representantes de la sociedad, haciendo conciencia, que la investigación es una oportunidad de desarrollo de la universidad, de la sociedad y del país, ya que todos son actores y beneficiarios de los resultados de la misma.

Es inminente trabajar en la preparación de los involucrados en el proceso de enseñanzaaprendizaje del inglés y otros de otros idiomas, que faciliten la gestión del conocimiento accediendo a insumos de información a nivel mundial y publicar a nivel internacional.

Entre las políticas de cambio debe incluirse el trabajo colaborativo entre docentes, estudiantes y autoridades, donde todos sean partícipes y generen compromiso con esta actividad. El primer paso sería la creación de un foro, donde expresen sus ideas, que sería el medio propicio para emprender el inicio de la generación de conocimiento, estas ideas deben enfocarse en las áreas críticas donde se requiere soluciones (investigación aplicada).

Se evidencia que las áreas de conocimiento en las que el Ecuador necesita generar investigación son en tecnología agroindustrial, educación, turismo y biotecnología.

El Estado debería establecer políticas públicas que faciliten las actividades de investigación colaborativa, a través de convenios a nivel gubernamental con otros países y con empresas privadas.

Por ende, hay que destacar que el rol que juega el Estado en este proceso es de vital importancia, sería trascendental que el mismo establezca procesos de conversaciones a nivel regional para la creación de un programa similar a Erasmus en Europa, en el ámbito de proyectos de investigación, para que los docentes y estudiantes se formen como investigadores en diferentes partes del mundo, adquieran diversidad de experiencias que puedan ser validados dichos conocimientos y experticia en cualquier país con los que se hayan firmado los acuerdos. 
Otras estrategias que se plantean son: la generación de convenios con instituciones internacionales para la cooperación y la investigación; estudios sobre líneas de investigación y ámbitos requeridos para dar soluciones locales a través de un conocimiento global; formación de redes internacionales que involucre a los miembros de la comunidad universitaria, generación de foros internacionales, organización de eventos y congresos internacionales con fines de divulgación de conocimientos de la comunidad científica internacional.

Tomando en cuenta que el Ecuador es un país diverso y que tiene muchos recursos interesantes para los investigadores extranjeros, se debe establecer como política de estado que todo investigador o estudiante de doctorado equivalente a Ph.D., que requiere hacer su investigación en el país, debe firmar convenio con una universidad e ir trabajando colaborativamente con la misma, para lo cual se debe hacer un listado de las universidades que estarían en capacidad de ofertar este servicio, en beneficio mutuo.

Uno de los criterios para que las universidades formen parte de este beneficio, debe ser la medición de esfuerzos que está haciendo la institución dentro del ámbito investigativo, como la formación de sus docentes y alumnos en idiomas extranjeros, la planificación de proyectos de investigación por áreas de conocimiento, por carreras o facultades, etc., y estar dentro de las primeras categorías de acuerdo a la categorización realizada a la universidad ecuatoriana por el CEAACES, es decir pertenecer a la "A" o "B". Este aspecto es muy importante, ya que las universidades se esforzarán en la mejora de su calidad en general y poner mucho énfasis en su "talón de Aquiles" que es la investigación.

\section{Estrategias para ampliar las alianzas con fines de investigación:}

- Establecer redes académicas de investigación a nivel nacional e internacional

- $\quad$ Establecer convenios específicos en temas de investigación

- Inclusión de la cátedra "metodología de la investigación" en el currículo de todas las carreras, pero entendida como método y no como sumatoria de técnicas.

- $\quad$ Suscripción a revistas técnico-científicas internacionales y a bibliotecas virtuales de universidades de prestigio o reconocidas por su excelencia académica, haciendo un seguimiento y medición de su uso en la IES.
- $\quad$ Apoyo técnico, económico y logístico a los investigadores para que realicen sus ponencias en eventos de divulgación científica nacionales e internacionales.

- Trabajar en la elaboración de revistas conjuntas con diversas universidades para su indexación.

- $\quad$ Difusión de la producción intelectual de los investigadores en medios de circulación masiva.

- Promover convenios con la Ciudad del Conocimiento Yachay, como centro tecnológico, ya que aglutinará a investigadores extranjeros, donde se pueden ejecutar investigaciones y proyectos colaborativos.

- Promover la realización de eventos académicos nacionales e internacionales con la participación de estudiantes y docentes para fomentar la investigación, así como eventos conjuntos entre universidades y con los sectores públicos y privados para identificar problemáticas existentes con necesidades de solución y plantear soluciones en conjunto, generando un compromiso en todas estas instancias.

\section{Indicadores para medir el impacto de la internacionalización de la universidad ecuatoriana en cuanto a proyectos de investigación y producción científica}

Toda acción en pro de la mejora de la calidad, debe ser medida, y la investigación no está exenta de esta acción, ya que da los parámetros para generar diagnósticos, y evidenciar avances o estancamientos en el ámbito de los proyectos de investigación. Es por tanto importante establecer indicadores que guíen hacia donde se dirige la investigación en el país y sobre todo si está generando impacto en el desarrollo.

Es importante establecer indicadores bibliométricos, de gestión, de personal, como algunos más que se exponen a continuación, que son valiosas herramientas para evaluar la actividad científica, siempre y cuando pueda ser complementada con el juicio de expertos, para que tenga mayor validez.

Para medir la actividad científica, también se deben establecer políticas por parte de cada una de las universidades, tanto para la planificación, gestión, como para la ejecución de la investigación y su evaluación.

A continuación se proponen algunos indicadores cuantitativos y cualitativos, para medir la actividad científica anual en la universidad ecuatoriana: 
- $\quad$ Número de docentes investigadores que publican con registros ISSN (International Standard Serial Number) e/o ISBN (International Standard Book Number), de acuerdo a su especialidad o área de conocimiento.

- Factor de impacto que tienen las publicaciones científicas de investigación (Journal Citation Reports)

- $\quad$ Porcentaje de docentes investigadores que participan en congresos o eventos académicos científicos internacionales.

- Número de afiliaciones a revistas científicas por parte de la universidad.

- Redes académicas nacionales e internacionales en las que colaboran los docentes de la universidad.

- Número de convenios en temas específicos de investigación.

- $\quad$ Presupuesto de apoyo para movilidad académica.

- Medios en los que se difunde la producción intelectual

- Proyectos colaborativos con IES especializadas o centros de investigación (institutos).

- Número de proyectos de investigación presentados oficialmente.

- $\quad$ Número de artículos indexados.

- Número de libros o capítulos de libros.

- Gestión de recursos en investigación

- $\quad$ Financiamiento externo

- $\quad$ Financiamiento local

- Investigadores por grado académico

- $\quad$ Investigadores por carrera

- Número de patentes y derechos de autor reconocidos por el Instituto Ecuatoriano de la Propiedad Intelectual (IEPI) o afines internacionales.

- $\quad$ Grupos de investigación y sus productos.

- $\quad$ Plan de investigación institucional.

- $\quad$ Autogestión para la investigación.

- Trasferencia de resultados y su vinculación.

\section{Prospectiva y resultados esperados:}

La prospectiva permite la valoración de las actividades de investigación, ya que lleva a proponer, prevenir, anticipar situaciones ahora, para tener un mejor futuro, por lo tanto es necesario resolver la pregunta de ¿Hacia dónde se quiere llegar con la investigación en la sociedad, en el país, y en la universidad ecuatoriana?.

La respuesta a la interrogante anterior es hacia el progreso, el bienestar y el desarrollo, esto se logrará con una visión de futuro polifacético y humanista (Mojica, 1991, p. 54), que se basa en la prospectiva mencionada por Berger (1964) sobre que el "[...] futuro no sucede ciega e irremediablemente, sino que depende de la acción del hombre [...]", es decir lo pone desde un punto de vista pragmático de que debe el ser humano dar inicio a la ejecución y no vienen las soluciones por inercia, sino que es la inteligencia humana la que genera el conocimiento, lo que requiere de la pro actividad, y capacidad de emprendimiento asegurando su presente, para poder enfrentar el futuro.

Al aplicar las estrategias propuestas se esperaría en primer lugar, un enriquecimiento en los procesos de generación de proyectos de investigación y producción científica, tanto en su calidad, como en su cantidad.

En segundo lugar, un mayor involucramiento y participación de los docentes y alumnos en los proceso de investigación; que las IES se involucren en una cultura de investigación, que aporten en el mejoramiento de la calidad de vida de la sociedad ecuatoriana, con proyección internacional, de tal manera que el Ecuador cuente con universidades de ranking mundial por investigación y por su calidad educativa.

Además se esperaría una transformación en la organización de la universidad, generando mayor creatividad, responsabilidad, autonomía y aporte por parte de sus actores, mayor exigencia en la preparación de los actores del pensamiento y gestores del conocimiento, es decir se esperaría un cambio en la cultura universitaria que albergue el espíritu investigativo de su claustro docente.

Se aspiraría a que la universidad pueda generar una sinergia con las entidades e instituciones que requieran ayuda técnica, para que así se cumpla el papel fundamental de la universidad que es la relación y generación de soluciones al entorno en el que se desenvuelve a través de estrategias de transferencia tecnológica y vinculación con la colectividad.

Para lograr que estos planteamientos prospectivos 
se vayan concretando es preciso que las universidades tomen en consideración, ciertas actividades como se propone a continuación:

- Empezar a concienciar a la comunidad académica, a las autoridades y los entes tomadores de decisiones sobre la importancia que tiene la investigación como actividad sustancial en la formación de los estudiantes y en la preparación del docente.

- $\quad$ Analizar sobre ¿Cuáles serán las fuentes de financiamiento para la investigación?, ya que en el caso de la universidad pública, no solo debe depender de los recursos escasos del Estado, sino buscar los medios financieros para el fomento de las actividades científicas de investigación, desde la elaboración de los planes, hasta la ejecución de los mismos, para lo cual deberán crear un área, departamento o unidad dedicada para el efecto.

- Incorporar en sus planificaciones estratégicas, dando la respectiva importancia a los proyectos de investigación y a la actividad científica, considerando que la internacionalización es un medio de crecimiento y que su ejecución permitirá un mejoramiento tanto en la calidad, como en la cantidad de producción científica.

- $\quad$ Incluir en el currículo de todas las carreras, la asignatura de metodología de la investigación científica.

- Reclutar nacional e internacionalmente a profesores investigadores de reconocida trayectoria investigativa que estén relacionados con las líneas de interés nacional.

- $\quad$ Aglutinar de portafolios de proyectos de investigación, para luego de un análisis, empezar a trabajarlos y propender su ejecución.

- $\quad$ Establecer mecanismos para la promoción de propuestas de proyectos de investigación y de esta manera obtener patrocinios.

La universidad ecuatoriana en el ámbito de proyectos de investigación y producción científica, debe llegar a promover la cultura de investigación, transferencia tecnológica y creatividad que potencie la excelencia académica de cada institución a nivel internacional basados en procesos eficientes, sistematizados y organizados capaces de generar recursos de tal manera que aporten con el conocimiento y soluciones a la comunidad local y global.

De esta manera preparar el camino hacia una prospectiva real y que pueda dar soluciones para un mejor desarrollo en las tres instancias planteadas que son la sociedad, el país y la universidad, que generen bienestar y desarrollo.

\section{CONCLUSIONES}

Cabe mencionar, después de lo expuesto, que el planteamiento de internacionalización para la universidad ecuatoriana en el ámbito de proyectos de investigación y producción científica, debe ser el eje de generación de conocimiento que proporcione soluciones a los múltiples requerimientos de la colectividad.

Para visibilizar la internacionalización y la mayor generación de producción científica y proyectos de investigación, es necesario que la universidad ecuatoriana incurra en actividades como participación en congresos, foros internacionales, redes de investigación; dinamización de sus páginas Web, con la investigación realizada, publicación y difusión de los resultados obtenidos en cada actividad científica.

Lograr que los resultados de las investigaciones gestionen el conocimiento para dar soluciones a los problemas de contaminación, y sobreexplotación de los recursos naturales del país, en beneficio de una sociedad mundial, como es el aprovechamiento de la biodiversidad y bioética.

Lo anterior se convierte en un atractivo para los investigadores internacionales y extranjeros, que aportarían con su contingente, con su conocimiento y experiencia en la generación de conocimiento y colaborarían en la preparación de los claustros académicos nacionales para que se proyecten internacionalmente con el producto de sus investigaciones científicas.

Es necesario pensar en el desarrollo y la internacionalización de la investigación porque es la que asegurará en prospectiva que los problemas del ahora tengan soluciones de innovación a futuro y de esta manera el Ecuador y sus sistema de educación superior a través de las universidades, formaría parte de este quehacer sustancial a nivel internacional, que es la investigación científica. 


\section{REFERENCIAS BIBLIOGRÁFICAS}

Altbach, P. G., \& Knight, J. (2007). The

internationalization of higher education: Motivations and realities. Journal of studies in international education, 11(3-4), 290-305.

Banco Mundial. (2014). Investigadores dedicados a investigación y desarrollo (por cada millón de personas). Retrieved from: http://datos. bancomundial.org/indicador/SP.POP.SCIE. RD.P6/countries?display=default

Beerkens, E., Brandenburg, U., Evers, N., van Gaalen, A., Leichsenring, H., \& Zimmerman, V. (2010). Indicator projects on internationalisation: Approaches, methods and findings.

Berger, G. (1964). Phénoménologie du temps et prospective.

Falagas, M. E., Kouranos, V. D., Arencibia-Jorge, R., \& Karageorgopoulos, D. E. (2008). Comparison of SClmago journal rank indicator with journal impact factor. The FASEB journal, 22(8), 2623-2628.

Ley Orgánica de Educación Superior, (2010). Mojica, F. (1991). La prospectiva. Técnicas para visualizar el futuro. Bogotá, Colômbia.

Romo Saltos, L. (2004). Universidad, Humanismo, Ciencia y Desarrollo. Quito, Ecuador: Editorial Casa de la Cultura Ecuatoriana.

UNESCO. (1998). DECLARACION MUNDIAL

SOBRE LA EDUCACION SUPERIOR EN EL SIGLO XXI: VISION Y ACCION. Retrieved from http://www.unesco.org/education/ educprog/wche/declaration_spa.htm declaracion

Vigil Taquechel, C. A. (2013). Aprendiendo de la experiencia del Programa ALBAN para mejorar la cooperación en materia de educación superior entre la Unión Europea y América Latina. Investigación \& Desarrollo, 21(1). 